\title{
Les urgences urologiques au centre hospitalier national de Nouakchott : aspects épidémiologiques, cliniques et thérapeutiques
}

\section{Urological emergencies at the national hospital centre of Nouakchott: epidemiological, clinical and therapeutic features}

\author{
Y. Ould Tfeil $\cdot$ C.A. Ould Elmoctar $\cdot$ M.O. Ca $\cdot$ C. Ould Jdoud \\ Reçu le 21 décembre 2009 ; accepté le 1 mars 2010 \\ (C) SALF et Springer-Verlag France 2010
}

Résumé But : Présenter les aspects épidémiologiques, cliniques et thérapeutiques des urgences urologiques en milieu africain.

Matériel et methods : Nous avons réalisé une étude rétrospective sur une période de cinq ans colligeant toutes les urgences urologiques reçues par l'équipe de garde au service d'urologie du centre hospitalier national de Nouakchott.

Résultats : Nous avons enregistré 1200 patients. L'âge moyen de nos patients était de 58,8 ans (un mois à 94 ans). Ces patients avaient un âge supérieur ou égal à 60 ans dans $50,7 \%$ des cas. Le rapport selon le sexe $(\mathrm{M} / \mathrm{F})$ était de 20,32. Les affections les plus fréquentes étaient la rétention aiguë d'urine (53\%) et les infections urogénitales qui représentaient dans leur ensemble 16,8\% des cas. Les gangrènes des organes génitaux externes représentaient 4,3\% des urgences et le priapisme 1,8\%. En urgence, 300 interventions chirurgicales ont été effectuées. Les principaux gestes réalisés étaient la pose d'un cystocathétérismesus-pubien $(59,7 \%)$ et le débridement d'une gangrène des organes génitaux externes + cystostomie de dérivation (16\%).

Conclusion: L'urgence urologique la plus fréquente dans notre pays était la rétention aiguë d'urine. Certaines pathologies graves, comme la gangrène des organes génitaux externes et le priapisme, n'y sont pas rares.

Mots clés Urgences urologiques · Épidémiologie · Clinique · Traitement $\cdot$ Nouakchott

Y. Ould Tfeil $(\bowtie) \cdot$ C.A. Ould Elmoctar · M.O. Ca ·

C. Ould Jdoud

Service d'urologie, centre hospitalier national de Nouakchott, 13,

Ksar, BP 612, Nouakchott, Mauritanie

e-mail : tefei12000@gmail.com
Abstract Objective: To present the epidemiological, clinical and therapeutic features of urological emergencies in Mauritania, West Africa.

Material and methods: The authors conducted a 5-year retrospective study that analyzed the epidemiological, clinical and therapeutic features of all urological emergencies admitted to the urology department of Nouakchott Central Hospital.

Results: There were 1,200 urological emergencies. The mean age of the patients was 58.8 years (range one month-94 years). These patients had an age $\geq 60$ years in $50.7 \%$ of the cases The sex ratio (M : F) was $20: 32$. The most frequent illnesses were urinary retention (53\%) and genitourinary system infections, which represented $16.8 \%$ of the cases. The gangrenes of male external genitalia (Fournier's gangrene) accounted for $4.3 \%$ of the cases and priapism $1.8 \%$. In emergency, 300 surgical operations were performed. The most performed procedures were the installation of a suprapubic catheter (59.7\%) and debridement of a gangrene of male external genitalia (16\%).

Conclusion: The most frequent urological emergency in our country was acute urinary retention. Serious illnesses, like gangrene of male external genitalia (Fournier's gangrene) and priapism, are not rare there.

Keywords Urological emergencies · Epidemiology · Clinical data $\cdot$ Treatment $\cdot$ Nouakchott

\section{Introduction}

Les urgences urologiques occupent une part importante mais souvent sous-estimée de l'activité de service d'urologie. Leur prise en charge dans un contexte largement dominé par l'activité réglée pose de nombreux problèmes. En Afrique, d'une manière générale, et en Mauritanie en particulier, bien que l'urgence soit un motif habituel 
d'hospitalisation dans les hôpitaux publics [1], les données sur l'épidémiologie des urgences urologiques sont rares.

Le but de notre étude était de présenter les aspects épidémiologiques et thérapeutiques des urgences urologiques en milieu africain.

\section{Matériel et méthodes}

Nous avons réalisé une étude rétrospective sur une période de cinq ans (janvier 2005 à décembre 2009) colligeant toutes les urgences reçues par l'équipe de garde au service d'urologie du centre hospitalier national de Nouakchott. L'équipe de garde était composée d'un ou de deux infirmiers, d'un médecin généraliste et d'un urologue en astreinte. Il existe au niveau du service une unité d'hospitalisation des urgences. Le médecin de garde assurait par ailleurs la surveillance des malades hospitalisés dans tout le service d'urologie, notamment les opérés récents.

Concernant les urgences urologiques, nous nous sommes intéressés à l'âge et au sexe des patients, au diagnostic, au nombre de malades hospitalisés et au type d'intervention chirurgicale réalisée en urgence. Le sondage urétral, bien que considéré par certains comme un acte médical, n'est pas un acte chirurgical. Les malades vus par le médecin de garde pour des pathologies urogénitales qui ne relèvent pas de l'urgence ou pour des urgences d'une autre spécialité (hernie inguinale étranglée, anurie non obstructive) ont été exclus de cette étude.

\section{Résultats}

Nous avons enregistré 1200 patients. L'âge moyen de nos patients était de 58,8 ans (un mois à 94 ans). Ces patients avaient un âge supérieur ou égal à 60 ans dans 50,7\% des cas. Le sex-ratio (M/F) était de 20,32. Les affections les plus fréquentes étaient la rétention aiguë d'urine $(53 \%)$ et les infections urogénitales qui représentaient dans leur ensemble $16,8 \%$ des cas (Tableau 1). Les principales étiologies des rétentions d'urine étaient les tumeurs prostatiques $(66,5 \%)$ et la sténose de l'urètre (23,3\%). Les gangrènes des organes génitaux externes représentaient $4,3 \%$ des urgences urologiques (Tableau 1), les traumatismes urogénitaux 3,8 \% et le priapisme $1,8 \%$. Parmi les traumatismes urogénitaux, les plus fréquents étaient le traumatisme fermé des bourses $(44,5 \%)$ et la rupture de l'urètre $(24,4 \%)$ (Tableau 2$)$. Le nombre d'hospitalisations en urgence était de 372 cas (31\%). En urgence, 300 interventions chirurgicales ont été effectuées. Les principaux gestes réalisés étaient la pose d'un cystocathétérisme sus-pubien $(59,7 \%)$ et le débridement d'une gangrène des organes génitaux externes + cystostomie de dérivation (16\%) (Tableau 3).

\begin{tabular}{|lll|}
\hline \multicolumn{2}{|l|}{ Tableau 1 Répartition des urgences urologiques } \\
\hline Pathologie & $\begin{array}{l}\text { Nombre } \\
\text { de cas }\end{array}$ & Pourcentage \\
\hline Rétention aiguë d'urine & 636 & 53 \\
Rétention chronique incomplète & 90 & 7,5 \\
$\quad$ d'urine & 140 & 11,67 \\
Infection urogénitale & 10 & 0,83 \\
Pyélonéphrite aiguë obstructive & 51 & 4,25 \\
Gangrène des organes génitaux & & \\
$\quad$ externes et du périnée & 82 & 6,83 \\
Hématurie totale & 71 & 5,92 \\
Colique néphrétique & 35 & 2,92 \\
Torsion du cordon spermatique & 21 & 1,75 \\
Priapisme & 45 & 3,75 \\
Traumatismes urogénitaux & 19 & 1,58 \\
Accidents de circoncision & & \\
\hline
\end{tabular}

Tableau 2 Répartition des traumatismes urogénitaux

\begin{tabular}{|lll|}
\hline Type de traumatisme & $\begin{array}{l}\text { Nombre de } \\
\text { cas }(\boldsymbol{n}=\mathbf{4 5})\end{array}$ & Pourcentage \\
\hline Traumatisme fermé du rein & 4 & 8,9 \\
Rupture de la vessie & 2 & 4,4 \\
Traumatisme fermé des bourses & 20 & 44,5 \\
Plaie scrotale & 6 & 13,4 \\
Rupture de l'urètre & 11 & 24,4 \\
Fracture de verge & 2 & 4,4 \\
\hline
\end{tabular}

\begin{tabular}{|lll|}
\hline Tableau 3 & Interventions chirurgicales réalisées en urgence \\
\hline Gestes & $\begin{array}{l}\text { Nombre de } \\
\text { cas }(\boldsymbol{n}=\mathbf{4 5})\end{array}$ & Pourcentage \\
\hline $\begin{array}{l}\text { Cystocathétérisme sus-pubien } \\
\text { Exploration + suture de plaie }\end{array}$ & 5 & 59,67 \\
$\quad$ scrotale & 37 & 1,67 \\
$\begin{array}{l}\text { Scrototomie exploratrice } \\
\text { Cure chirurgicale de priapisme }\end{array}$ & 21 & 12,33 \\
$\begin{array}{l}\text { Débridement + cystostomie } \\
\text { Néphrostomie de dérivation }\end{array}$ & 48 & 16 \\
\hline
\end{tabular}

\section{Discussion}

La rétention aiguë d'urine est l'urgence urologique la plus fréquente dans notre pays. Cela s'explique par le fait qu'elle y constitue l'une des principales circonstances de découverte des tumeurs prostatiques et de la sténose de l'urètre. En effet, pour des raisons socio-économiques et culturelles, la plupart des personnes porteuses de ces 
pathologies ne consultent qu'à la phase de complications. L'âge moyen de nos patients était de 58,8 ans, et 50,7\% d'entre eux avaient un âge supérieur ou égal à 60 ans. En Espagne, Parra Muntaner et al. [2] ont trouvé un âge moyen de 53 ans et une plus grande incidence des urgences urologiques chez les hommes de plus de 60 ans. Cependant, dans les pays européens, la rétention aiguë d'urine n'est pas l'urgence urologique la plus fréquente. Elle représentait $22 \%$ des motifs de consultations en France [3], tandis que son incidence annuelle était de 3,06/1 000 en Angleterre [4]. Les principales étiologies de la rétention d'urine dans cette étude étaient les tumeurs prostatiques et la sténose de l'urètre. Ces résultats sont comparables à ceux de Ikuerowo et al. [5] au Nigeria, où l'hypertrophie bénigne de la prostate $(64 \%)$ et la sténose de l'urètre $(28,4 \%)$ sont les principales étiologies de la rétention aiguë d'urine. Cette très grande incidence de la rétention aiguë d'urines dans notre pays engendre de nombreux problèmes. En effet, la plupart de ces patients vont nécessiter un traitement chirurgical et seront obligés de patienter des mois dans l'attente de ce traitement. Il en résulte de longues durées de port de sonde urinaire à demeure, durées pendant lesquelles les patients sont exposés à des complications infectieuses en plus de la détérioration de leur qualité de vie et du coût économique de cette affection $[5,6]$.

Les infections urogénitales représentaient dans leur ensemble $16,8 \%$ des cas. Leurs particularités dans notre étude étaient la fréquence des gangrènes des organes génitaux externes et du périnée $(4,3 \%)$ et la rareté des pyélonéphrites aiguës obstructives $(0,8 \%)$. Les gangrènes des organes génitaux externes rencontrées ici sont essentiellement des complications d'une sténose de l'urètre. Le diagnostic était souvent tardif à un stade où l'extension locorégionale des lésions était importante (Fig. 1). Le traitement reposait sur une bi- ou triantibiothérapie et sur un débridement chirurgical éventuellement répété, excisant toutes les zones nécrosées. Une cystostomie était presque systématiquement associée au débridement chirurgical. Les gangrènes des organes génitaux externes n'ont pas été rapportées dans la plupart des études européennes sur les urgences urologiques $[2,3,7]$. Ces gangrènes des organes génitaux externes et les rétentions d'urine dues, dans $23,3 \%$, à la sténose de l'urètre font de cette sténose de l'urètre la deuxième pourvoyeuse d'urgences urologiques dans notre pays après les tumeurs prostatiques. Le drainage en urgence des pyélonéphrites aiguës obstructives ne représentait que 3,4\% des interventions chirurgicales réalisées dans cette étude, alors que dans l'étude de Mondet et al. [3] en France, elle constituait $31 \%$ des interventions. Ce drainage consistait dans tous les cas à une néphrostomie de dérivation. La montée de sonde JJ n'a pas été réalisée ici faute d'équipements adéquats. Nous pensons que la fréquence réelle de ces pyélonéphrites aiguës obstructives dans notre pays est beaucoup plus importante, vu la fréquence des pathologies pouvant entraîner une obstruction de la voie excrétrice supérieure comme la lithiase urétérale et les séquelles de bilharziose ou de tuberculose urogénitale. Par ailleurs, la plupart des patients hospitalisés pour colique néphrétique fébrile et traités par la suite par néphrostomie de dérivation l'ont été dans les 48 à 72 heures. Ces dernières interventions n'ont donc pas été prises en compte dans l'activité de l'équipe de garde.

Les traumatismes des organes génitaux externes étaient les traumatismes urogénitaux les plus fréquents. Ils étaient le plus souvent bénins et survenaient lors des rixes ou comme accidents de sport. Une scrototomie exploratrice a été effectuée dans 37 cas $(12,4 \%)$. Pour des traumatismes plus violents (accidents de la voie publique), Paparel et al. [8] ont trouvé que les reins (43\%) et les testicules $(24 \%)$ étaient les organes urogénitaux les plus atteints. Les accidents de circoncision sont fréquents dans notre service $[9,10]$ à cause de l'effet centre. En effet, ces complications font suite à des circoncisions réalisées par des tradipraticiens ou des agents paramédicaux en dehors du service. En urgence, il s'agissait souvent de complications à type d'hémorragie minime mais persistante, de surinfection de la plaie, d'amputation totale ou partielle du gland (deux cas), ou de rétention d'urine.

\section{Conclusion}

La grande fréquence des rétentions aiguës d'urine, bien sûr sur obstacle prostatique, mais aussi sur sténose urétrale, le traitement d'urgence devenant alors spécifiquement urologique, la nécessité de drainer en urgence les pyélonéphrites obstructives plutôt que tardivement et la gravité des

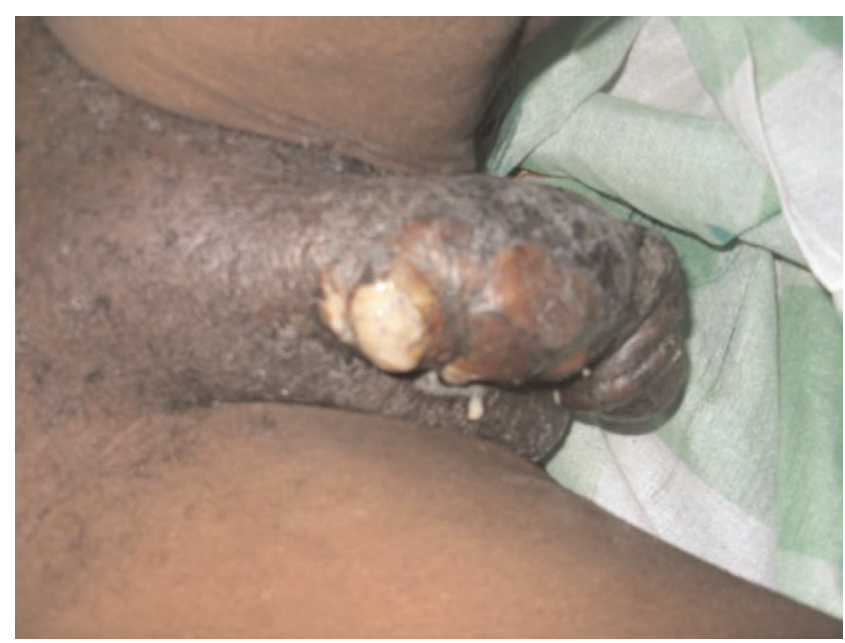

Fig. 1 Gangrène des organes génitaux externes 
priapismes qui ont justifié un nombre important d'interventions d'urgence font que la création d'une garde propre à la spécialité est une option raisonnable dans l'organisation du système de soins de notre pays.

\section{Conflit d'intérêt : aucun.}

\section{Références}

1. Zué AS, Josseaume A, Nsafu DN, et al (2003) Les urgences chirurgicales au centre hospitalier de Libreville. Ann Fr Anesth Reanim 22: 189-95

2. Parra Muntaner L, López Pacios JC, Piñeiro Fernández MC, et al (2001) Urologic disease emergency: clinico-epidemiolgic analysis at a district hospital. Arch Esp Urol 54: 411-5
3. Mondet F, Chartier-Kastler E, Yonneau L, et al (2002) Épidémiologie des urgences urologiques en centre hospitalier universitaire. Prog Urol 12: 437-42

4. Cathcart P, van der Meulen J, Armitage J, Emberton M (2006) Incidence of primary and recurrent acute urinary retention between 1998 and 2003 in England. J Urol 176: 200-4

5. Ikuerowo SO, Ogunade AA, Ogunlowo TO, et al (2007) The burden of prolonged indwelling catheter after acute urinary retention in Ikeja-Lagos, Nigeria. BMC Urol 7: 16

6. Thomas K, Oades G, Taylor-Hay C, Kirby RS (2005) Acute urinary retention: what is the impact's on patient quality of life? BJU Int 95: 72-6.

7. Danuser H, Ackermann DK, Studer UE (1993) Urological emergencies. Schweiz Med Wochenschr 123(15): 749-55

8. Paparel P, N'Diaye A, Laumon B, et al (2006) The epidemiology of trauma of the genitourinary system after traffic accidents: analysis of a register of over 43,000 victims. BJU Int 97: 338-41

9. Sylla C, Diao B, Diallo AB, et al (2003) Les complications de la circoncision. À propos de 63 cas. Prog Urol 13: 266-72

10. Ba M, Ndoye A, Fall PA, et al (2000) Les fistules péniennes après circoncision : à propos de 20 observations. Andrologie 10: 87-115

\section{旬 Springer}

the language of science

\section{springer.com}

\section{The Innovative Website} Focused on You

- Sign up for SpringerAlerts to get the latest news in your field

- Save money through Springer's Online Sales

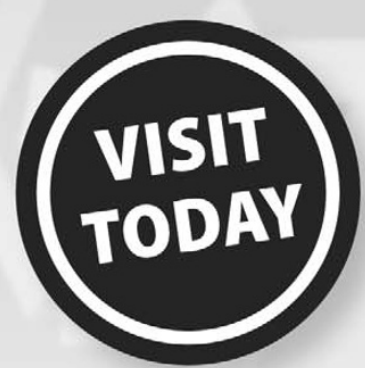

- Order with special savings - for authors, journal contributors, society members and instructors

- Find all books and journals

- Download free e-sample copies of journals and book chapters

\section{springer.com - be the first to know}

